\title{
AMANDA: An Autonomous Self-Powered Miniaturized Smart Sensing Embedded System
}

\author{
Charalampos S. Kouzinopoulos, Dimitrios Tzovaras \\ Information Technologies Institute \\ Centre for Research and Technology Hellas \\ Thessaloniki, Greece \\ \{kouzinopoulos, dimitrios.tzovaras\}@iti.gr \\ Mathieu Bellanger, Matthias Kauer \\ Lightricity Limited \\ Oxford, United Kingdom \\ \{mathieu.bellanger, matthias.kauer\}@ lightricity.co.uk \\ Martin Schellenberg \\ Microdul AG \\ Zurich, Switzerland \\ martin.schellenberg@microdul.com
}

\author{
Pawel Bembnowicz \\ Stichting IMEC Nederland \\ pawel.bembnowicz@imec.nl
}

Eindhoven, The Netherlands Zurich University of Applied Sciences

Zurich, Switzerland

mema@zhaw.ch

\author{
Denis Pasero \\ Ilika Technologies Ltd \\ Romsey, United Kingdom \\ denis.pasero@ilika.com
}

\author{
Oskar Vujicic \\ Penta d.o.o. \\ Pula, Croatia \\ oskar.vujicic@penta.hr
}

\begin{abstract}
This paper introduces an Autonomous Smart Sensing Card (ASSC), an embedded system that will be powered indoors and outdoors by harvested energy, have miniaturized dimensions and serve multi-sensorial IoT applications for smart living and working environments. It will consist of a combination of newly developed and optimized off-the-shelf or close-tocommercialization technologies such as PV harvesters, energy storage and power management units, MCUs and sensors, all packed with a form factor under $3 \mathrm{~mm}$ in thickness. The system will introduce technical breakthroughs that will boost further miniaturization, a small footprint, ultra-low power consumption as well as short- and long-range communications.

Index Terms-Electronic smart systems, Embedded systems, Sensors, Microelectronics, Miniaturization, Autonomous operation, Energy harvesting
\end{abstract}

\section{INTRODUCTION}

Electronic Smart Systems (ESS) are embedded miniaturized electronics that can be used for both sensing and actuating with a focus on autonomous operation, energy efficiency and networking capabilities as well as adaptation, reliability, and security. The global ESS market was valued at USD 30.82 billion in 2018, and is estimated to reach USD 85.93 billion by the end of 2024, with a compound annual growth rate of $18.82 \%$ during the period of $2019-2024$ [1]. The next phase of digital transformation has arrived, and it reaches to the far edges of the networks, with connections to billions of devices and objects collecting and transmitting data from ever-evolving sensors.

Smart sensors have evolved from being discrete devices designed to detect specific properties (e.g. light, heat, motion), to fully integrated sensor systems, offering enhanced computing capabilities, connected within any number of autonomous sys- tems such as wearable technologies and smart living environments. There has been a steady shift towards miniaturization of ESS, using micro- or nano-electromechanical technology, bringing new concepts and technologies that will benefit the environment of smart cities, such as air quality, temperature, humidity, noise and occupancy monitoring as well as security of people or assets, including imaging, tracking, fingerprint, data privacy and cyber-security.

Despite the fact that there are many academic, research and industrial entities in Europe with state-of-the-art competency and infrastructures to promote innovations in this area, there are still technological gaps to be covered, in terms of size, cost, materials, design and manufacturing processes of ESSs. In order to address these issues, this paper proposes a unique ASSC which will have the size, feel and look of a credit card, ideal for easy deployment outdoors, in buildings or as a wearable. Any technological challenges will be overcome through the optimization of connectivity, miniaturization, power consumption, security, intelligence, design and cost. A combination of photovoltaic (PV) components, energy storage and power management units as well as sensors will be developed and optimized for the AMANDA ASSC and integrated with existing off-the-shelf components in a single system less than $3 \mathrm{~mm}$ thick, including packaging. The final design will target a large number of applications across different sectors, such as smart irrigation (agriculture), predictive and adaptive building environmental control (smart cities), asset tracking (industrial) as well as identification card, weather monitoring station and health monitoring device (consumer electronics). AMANDA will introduce technical breakthroughs that will boost further miniaturization, offer a small footprint and ultra-low power 


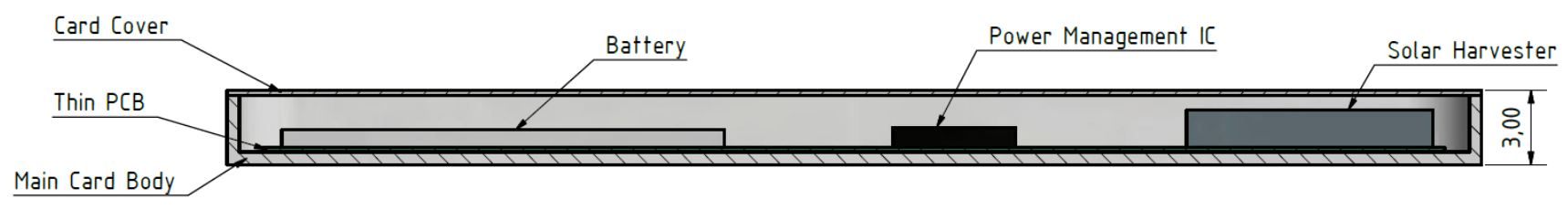

Fig. 1. Cross section of the AMANDA ASSC

consumption allowing for both indoors and outdoors operation, with a projected lifetime of over 10 years.

This paper is structured as follows: Section II provides a literature review on Autonomous Self-Powered Miniaturized Smart Sensing Embedded Systems and on individual sensor, processing and energy-related components. Section III presents the architecture of the introduced ASSC, highlighting the current status of the components developed and optimized for the AMANDA ASSC and a number of target goals. Finally, Section IV draws the conclusions of this paper and discusses future work.

\section{RELATED WORK}

The concept of miniaturized autonomous systems is not new. EcoMicro [2] is a miniaturized 9-Degrees of Freedom inertial-sensing node, with short-range wireless communication features and energy autonomy, utilizing energy storage and solar cells. The main advantage of this device over the state-of-the-art designs is its miniature size, with a volume of $8 \times 13 \times 9.5 \mathrm{~mm}$ while at the same time providing motion measurement, via an Inertia Measurement Unit (IMU) and data transmission over Bluetooth Low Energy (BLE). A mechanically flexible and fully integrated wearable sensor array was demonstrated in [3] for in situ perspiration analysis. It measures sweat metabolites and electrolytes, as well as skin temperature, to calibrate the response of the sensors. The system processes data on-board and communicates using BLE. It has no energy harvesting. The Mobile Sensing Platform [4] is a small wearable device which utilizes different sensors, such as microphones, light sensors, 3-axis digital accelerometers, barometers, thermometers, humidity sensors and digital compasses to provide useful data for activity-aware systems. Although small in dimensions, the device has a considerable thickness. Yang, Xu, Vervust and Vanfleteren [5] describe a miniaturized sensor patch that integrates all necessary readout electronics capable of simultaneous impedance and temperature measurements. The state of the art is summarized in Table I. The systems mentioned above however are very limited as far as their use in IoT environments is considered. They do not integrate long-range wireless communication, capable of enhancing mobility. Such systems are more demanding in terms of energy and thus require more space for the energy harvester. The type of solar harvester that [2] uses, limits it to outdoor operation where there is enough light. Furthermore, components chosen such as the power management unit (PMIC) and the BLE radio are not the best and leave room for improvements in the energy consumption.

TABLE I

SUMMARY OF THE STATE OF THE ART

\begin{tabular}{|c|c|c|c|c|}
\hline & EcoMicro & $\begin{array}{l}\text { Fully } \\
\text { integrated } \\
\text { wearable } \\
\text { sensor }\end{array}$ & $\begin{array}{l}\text { Mobile } \\
\text { Sensing } \\
\text { Platform }\end{array}$ & $\begin{array}{l}\text { Miniaturized } \\
\text { sensor } \\
\text { patch }\end{array}$ \\
\hline Sensing & $\begin{array}{l}\text { Accelerometer, } \\
\text { compass, } \\
\text { gyroscope }\end{array}$ & $\begin{array}{l}\text { Wearable } \\
\text { FISA for } \\
\text { simulta- } \\
\text { neous and } \\
\text { selective } \\
\text { screening of } \\
\text { a panel of } \\
\text { biomarkers } \\
\text { in sweat }\end{array}$ & $\begin{array}{l}\text { Microphone, } \\
\text { accelerom- } \\
\text { eter, light, } \\
\text { temper- } \\
\text { ature, } \\
\text { humidity, } \\
\text { compass }\end{array}$ & $\begin{array}{l}\text { In situ, } \\
\text { continuous } \\
\text { impedance } \\
\text { and } \\
\text { temperature } \\
\text { measure- } \\
\text { ment }\end{array}$ \\
\hline $\begin{array}{l}\text { Harvesting } \\
\text { and Power } \\
\text { manage- } \\
\text { ment }\end{array}$ & $\begin{array}{l}2 \text { on-board } \\
\text { miniature } \\
\text { solar cells } \\
\text { with MPPT }\end{array}$ & - & $\begin{array}{l}\text { No } \\
\text { harvesting, } \\
1800 m A h \\
\text { energy } \\
\text { storage }\end{array}$ & $\begin{array}{l}\text { No power } \\
\text { man- } \\
\text { agement } \\
\text { carried out }\end{array}$ \\
\hline $\begin{array}{l}\text { Short- } \\
\text { range } \\
\text { wireless }\end{array}$ & BLE & Bluetooth & $\begin{array}{l}\text { Bluetooth / } \\
\text { Zigbee }\end{array}$ & - \\
\hline $\begin{array}{l}\text { Long- } \\
\text { range } \\
\text { wireless }\end{array}$ & - & - & - & - \\
\hline $\begin{array}{l}\text { Indoors / } \\
\text { outdoors } \\
\text { operation }\end{array}$ & $\begin{array}{l}\mathrm{EH} \text { but } \\
\text { requirement } \\
\text { for outdoors }\end{array}$ & Both & $\begin{array}{l}\text { Both, } \\
\text { thanks to } \\
\text { energy } \\
\text { storage }\end{array}$ & Both \\
\hline Dimensions & $\begin{array}{l}8 \times 13 \times \\
9.5 \mathrm{~mm}\end{array}$ & & $\begin{array}{l}5.08 \times \\
3.56 \mathrm{~mm}\end{array}$ & $21 \times 24 \mathrm{~mm}$ \\
\hline $\begin{array}{l}\text { Extra Fea- } \\
\text { tures }\end{array}$ & RTC & $\begin{array}{l}\text { Wearable } \\
\text { application }\end{array}$ & $\begin{array}{l}\text { MiniSD } \\
\text { card slot }\end{array}$ & \\
\hline
\end{tabular}

The distinguishing feature of the AMANDA ASSC is the integration of multiple low-power methods and techniques within a miniaturized size factor where the thickness restriction of $3 \mathrm{~mm}$ applies to all components, including energy storage and environmental sensors. The starting point is the ultra-low power approach in design, with the system capable of switching various components completely off, as demonstrated in [6]. This method allows the elimination of leakage sources, especially at higher temperatures where leakages are not negligible. In this way, it is possible to totally cut off the microcontroller, sensors and wireless systems, leaving only the key monitoring elements active. This enables consumption down to tens/hundreds of nano-ampere for the whole temperature range in the lowest power mode, where many applications will 
spend most of the time. In that mode, a very low-power and yet accurate Real-time clock (RTC) is utilized to keep time and trigger system restarts regularly, giving great flexibility for energy optimization. An illumination of 20 lux should be enough to compensate for the consumption of the RTC and the capacitive sensing systems through the PV cells and the use of a dedicated power management subsystem.

The system also uses Ferroelectric RAM (FRAM) for both parameters and data storage. Contrary to Flash or EEPROM, FRAM allows low-power access and non-volatile operations over tens of years, since it can be rewritten billions of times. This greatly enhances energy consumption and allows all status registers to be stored in FRAM with minimum energy. It is possible to switch the platform off without losing the status of the micro-controller unit (MCU) [7], [8] or data stored in FRAM. This enhancement also massively improves the reliability of the system, since it provides a low-power method for storing and recovering data, a vital factor for systems powered by harvested energy.

In terms of the form factor, having an on-board low-power $\mathrm{CO}^{2}$ concentration sensor at such scale as well as ultra-thin solid state batteries and miniaturized solar harvesters with surface areas measuring a few $\mathrm{mm}^{2}$ has, to the best of our knowledge, not been done before. Finally, the device also improves on the state-of-the-art low-power, energy-harvesting sensing platforms by utilizing a combination of two different wireless protocols, allowing for data transmission both in short and long ranges via BLE and Long Range wireless (LoRa/LoRaWAN) accordingly [9]. The BLE radio chosen is currently the best on the market, according to [10], allowing for a non-negligible reduction of energy consumption.

\section{ThE AMANDA ARCHITECTURE}

The AMANDA ASSC will have a minimal form factor, including all the essential components for its operation: a photovoltaic energy harvester from Lightricity; a miniaturized solid-state energy storage unit from Ilika; an MCU and PMIC from E-peas; a capacitive sensor from Microdul for humandevice interaction; two wireless communication units (RF); an RTC; a nano-timer and a non-volatile memory unit. Depending on the usage scenario, the ASSC will also integrate: a temperature sensor from Microdul; a $\mathrm{CO}_{2}$ sensor from IMEC; and an image sensor from E-peas. A number of additional offthe-shelf sensors will also be included, although details have not been finalized at this stage of development.

\section{A. Form factor}

The target dimensions of the ASSC are $89 \times 51 \mathrm{~mm}$, the US standard size for business cards. The maximum thickness of the system will be constrained to $3 \mathrm{~mm}$. A cross section of the final system is given in Figure 1. There are a few layered components that need to fit vertically:

- Main card body. This part will house the printed circuit board (PCB) and will be used as the device base. Depending on the material and the manufacturing process used, a minimum thickness, estimated at $0.5 \mathrm{~mm}$, will be applied

- Thin PCB. This part will accommodate all electronic components used for the card and will cover the majority of the available surface. Depending on the type of board that will be used, such as a flexible PCB or a thin FR4 board, a minimum thickness will also be applied. Based on state-of-the art thickness for flexible PCBs, a range between $0.2 \mathrm{~mm}$ and $0.4 \mathrm{~mm}$ is feasible, depending on the number of layers of the board

- Card cover. To enclose all components inside the card base, different types of covers are considered. As seen on similar designs of electronic smart cards, a solution based on a plastic sticker cover could be used, with an additional thickness of approximately $0.2 \mathrm{~mm}$

\section{B. System components}

Figure 2 depicts a conceptual view of the individual component placement on the AMANDA ASSC. Most of the components have a working version, denoted as current, while improved versions, referred to as target, are under development for the ASSC.

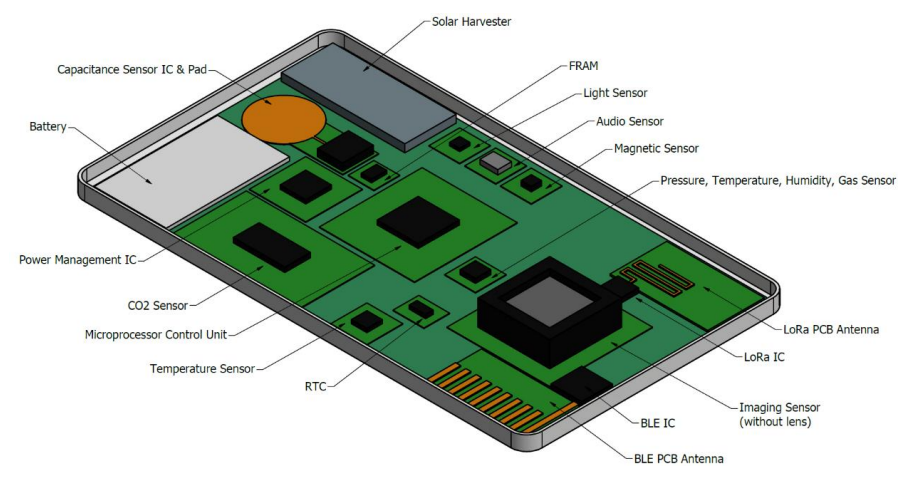

Fig. 2. Conceptual component placement on the AMANDA ASSC

1) Energy harvester: The energy harvester will supply continuous power to the AMANDA ASSC. The amount of provided power will mainly depend on the illumination level of the environment and on whether the ASSC is moving or is stationary. The electrical performance of the harvester for 200 lux is shown in Table II. Although the performance of the harvester may be slightly improved for the ASSC, the key focus will be to develop a surface-mount technology (SMT)compatible device that can withstand a solder re-flow process (approximately $250{ }^{\circ} \mathrm{C}$ ).

TABLE II

ELECTRICAL SPECIFICATIONS OF THE ENERGY HARVESTER

\begin{tabular}{|c|c|c|}
\hline Performance at 200 lux & Current & Target \\
\hline Output voltage (MPP) & $1 \mathrm{~V}$ & $>1 \mathrm{~V}$ \\
\hline Output current (MPP) & $50 \mu \mathrm{A}$ & $>50 \mu \mathrm{A}$ \\
\hline Efficiency & $30 \%$ & $>30 \%$ \\
\hline PV active area & $2.15 \mathrm{~cm}^{2}$ & $<2.15 \mathrm{~cm}^{2}$ \\
\hline
\end{tabular}


As detailed in Table III, the external dimensions of the harvester are currently $2.4 \times 1.0 \mathrm{~cm}$. These dimensions may be reduced down to $<1 \mathrm{~cm}^{2}$ in the future, for versions of the ASSC with lower load requirements. To enable these targets, the focus will be on new device packaging processes that can achieve lower thickness and better surface finish. Reducing thermal stress during the packaging process will be important for achieving the target thickness of $<1 \mathrm{~mm}$.

TABLE III

MECHANICAL SPECIFICATIONS OF THE ENERGY HARVESTER

\begin{tabular}{|c|c|c|}
\hline Geometrical parameters & Current & Target \\
\hline Outer module dimensions & $2.4 \times 1.0 \mathrm{~cm}$ & $\leq 2.4 \times 1.0 \mathrm{~cm}$ \\
\hline Thickness & $>1.5 \mathrm{~mm}$ & $<1 \mathrm{~mm}$ \\
\hline
\end{tabular}

2) Energy storage: The role of the solid-state energy storage (ES) is to store energy provided by the energy harvester and deliver it on-demand to the rest of the ASSC, including the MCU and sensors. During periods of illumination, the PV cell will charge the ES and power the device; during dark periods, the ES will discharge into the rest of the circuitry, hence ensuring that the ASSC can operate under all light levels.

TABLE IV

ELECTRICAL SPECIFICATIONS OF THE ENERGY STORAGE

\begin{tabular}{|c|c|c|}
\hline Energy storage & Current & Target \\
\hline Rated Capacity & $0.25 m A h$ & $2 m A h$ \\
\hline Voltage & $3-4 V$ & $3-4 V$ \\
\hline Standard continuous discharge current & $0.25 m A$ & $2 m A$ \\
\hline Peak current $(<0.5 m s$ pulse $)$ & $5 m A$ & $40 m A$ \\
\hline Storage footprint & $40 \%$ & $80 \%$ \\
\hline
\end{tabular}

The ES under development for the AMANDA ASSC will use improved manufacturing techniques which will significantly increase the footprint efficiency and therefore the capacity of the storage without compromising the cycle life and performance. The targeted final ES will have a nominal voltage of $3.5 \mathrm{~V}$ with a target capacity of $2 \mathrm{mAh}$ giving a total of $7 \mathrm{mWh}$ of stored energy to drive the ASSC. The target specification of the ES would enable a maximum continuous load of $20 \mathrm{~mA}$ and a short duration pulsed load of $40 \mathrm{~mA}$.

3) $M C U$ : The E-peas general purpose micro-controller series is dedicated to sensing applications. It is based on the ARM Cortex-M0 32-bit architecture with a $24 \mathrm{MHz}$ operating frequency and a very low power consumption, both in active and standby mode. The specifications of the MCU are summarized in Table $\mathrm{V}$. The active current of the MCU is $29 \mu \mathrm{A} / \mathrm{MHz}$ while the current in Deepsleep with an RTC running and an $8 k B$ SRAM retention is $850 n A$. The MCU currently has up to $256 \mathrm{kB}$ of embedded non-volatile singlecycle flash memory with an instruction cache to reduce powerhungry flash accesses and up to $32 k B$ of embedded SRAM memory with SRAM retention available per $8 k B$ bank in Deepsleep.

The system has a DMA controller with 8 channels and inter-peripheral signaling, for core-less memory transfer and peripheral communication. It has clock gating and scaling available for each peripheral individually. The communication is performed with one UART with Deepsleep support. Two master/slave SPIs exist with multi-master support, up to 4 chip select, 8-byte FIFO and DMA support. Two master/slave $\mathrm{I}^{2} \mathrm{C}$ are also present, with multi-master support, 7- and 10-bit addressing modes, up to FM+ $(1 \mathrm{Mbit} / \mathrm{s})$ and DMA support. Finally there up to 48 GPIOs available. On the MCU there are four 32-bit multi-function timers with two compare/capture channels for PWM generation, edge counting or frequency measurement and DMA support. Additionally, there is one RTC with programmable periodic interrupt generation and Deepsleep support as well as one 32-bit Watchdog with Deepsleep support.

In order to store multiple sensor firmware and to be able to process the more complex sensor data, the MCU for the AMANDA ASSC will have a larger memory, with at least $256 \mathrm{kB}$ of Flash and at least $256 \mathrm{kB}$ of SRAM. Furthermore, a core architecture with increased processing capabilities will be required in order to minimize the active time and the energy consumption.

TABLE V

SPECIFICATIONS OF THE MCU

\begin{tabular}{|c|c|c|}
\hline MCU & Current & Target \\
\hline Voltage supply & $1.8-3.3 \mathrm{~V}$ & $1.8-3.3 \mathrm{~V}$ \\
\hline Active current & $29 \mu \mathrm{A} / \mathrm{MHz}$ & $<20 \mu \mathrm{A} / \mathrm{MHz}$ \\
\hline Deepsleep current & $850 \mathrm{nA}$ & $<500 \mathrm{nA}$ \\
\hline Sleep wake-up time & 1 clock cycle & 1 clock cycle \\
\hline Deep sleep wake-up time & Approximately $1 \mu \mathrm{s}$ & Approximately $1 \mu \mathrm{s}$ \\
\hline
\end{tabular}

4) PMIC: The PMIC is an integrated energy management circuit that extracts DC power from up to 4-cell solar panels to simultaneously store energy in a rechargeable element and supply the system with two independent regulated voltages. The PMIC harvests the available input current up to $110 \mathrm{~mA}$, integrating an ultra-low power boost converter. The quiescent current of the PMIC with the Low-dropout regulators (LDOs) enabled is $600 n A$. The boost converter operates with input voltages in a range between $50 \mathrm{mV}$ to $5 \mathrm{~V}$. Supplied with a cold-start circuit, it can start operating with empty storage elements at an input voltage as low as $380 \mathrm{mV}$ and an input power of $3 \mu \mathrm{W}$. The low-voltage supply can typically drive a micro-controller at $1.2 \mathrm{~V}$ or $1.8 \mathrm{~V}$. The high-voltage supply typically drives a radio transceiver at a configurable voltage between $1.8 \mathrm{~V}$ and $4.1 \mathrm{~V}$. Both are driven by LDOs for low noise and high stability. The PMIC also integrates a maximum power point tracking (MPPT) module that allows for the maintenance of the PV cell at its optimal operation point.

A target for the PMIC is to reduce its quiescent current down to $200 n A$ while further including a dedicated sleep mode. While in sleep mode, the load outputs will be shut down and the energy storage will be disconnected from the PMIC. The storage will only be connected periodically if energy harvesting occurs, to keep recharging it. The DC/DC converter operates with input voltages in a range from $50 \mathrm{mV}$ 
to $4 V$. The cold-start circuit will be improved so that the PMIC will start operating with an empty storage element from an input voltage as low as $300 \mathrm{mV}$ and an input power of $2 \mu \mathrm{W}$. The PMIC will provide two regulated output voltages, thanks to a combination of $\mathrm{DC} / \mathrm{DC}$ regulators. These voltage levels can be programmed, with their default value being $1.8 \mathrm{~V}$ and $3.3 \mathrm{~V}$. The DC/DC converters can be disabled or enabled by the micro-controller. The PMIC is available in a QFN28 $5 \times 5 \mathrm{~mm}$ package. The complete system fits in less than $1 \mathrm{~cm}^{2}$ including all the required passive components.

5) Capacitive sensor: The capacitive sensor will be used to wake up the ASSC from stand-by. The sensor will be based on MS8891 [11], with an active power consumption of approximately $750 \mathrm{nA}$. MS8891 can be operated in three essentially different modes: Op-mode-1, with an on demand absolute capacitance measurement, Op-mode- 2 with a periodic self-timed (2/second, 32/second, permanent) touch detection as well as Op-mode-3 with an on demand touch detection.

A further reduction in the power consumption of MS8891 for the autonomous periodic measuring mode (Op-mode-2) will be targeted for the AMANDA ASSC. A power reduction in Op-mode-2 directly translates to longer energy storage life in dark conditions where little to no energy can be harvested. The improvements targeted for the capacitive sensor include the implementation of auto-calibration of the switching threshold in Op-mode-2 and Op-mode-3, the reduction of power consumption in periodic Op-mode- 2 by reducing idle supply current with oscillator enabled as well as the improvement of noise immunity if required and if power consumption is not increasing.

6) Temperature sensor: The temperature sensor is a fully integrated and calibrated solid-state silicon sensor, based on the well-known temperature characteristic of the silicon diode forward voltage. It has a typical temperature measurement accuracy of $+/-0.3{ }^{\circ} \mathrm{C}$. It is available in either a $3 \times 3 \mathrm{~mm}$ QFN16 package or a chip-scale package package. Table VI presents the current and target energy specifications of the sensor.

TABLE VI

ENERGY SPECIFICATIONS OF THE TEMPERATURE SENSOR (TYPICAL VALUES)

\begin{tabular}{|c|c|c|}
\hline Temperature sensor & Current & Target \\
\hline $\mathrm{V}_{\text {supply }}$ & $3.0 \mathrm{~V}$ & $1.8 \mathrm{~V}$ \\
\hline $\mathrm{E}_{\text {start-up }}$ & $0.6 \mu \mathrm{J}$ & $0.36 \mu \mathrm{J}$ \\
\hline $\mathrm{E}_{\text {measurement }}$ & $11.3 \mu \mathrm{J}$ & $3.8 \mu \mathrm{J}$ \\
\hline $\mathrm{I}_{\text {dd,average }}$ & $80 \mathrm{nA}$ & $40 \mathrm{nA}$ \\
\hline
\end{tabular}

7) $\mathrm{CO}_{2}$ sensor: $\mathrm{A} \mathrm{CO}_{2}$ transducer and dedicated electronic readout system. It consists of an interdigitated electrode on top of which a layer of gas-sensitive material is drop casted. Gas diffuses into the $\mathrm{CO}_{2}$ sensitive layer and changes its electrical property. The change can be read out as a change of electric impedance between interdigitated electrodes. Currently, the sensor has a sensitivity of about $1.2 \Omega / p p m \mathrm{CO}_{2}$ at $1 \mathrm{~Hz}$ measurement excitation. The expected range of the sensor's measurement is $\operatorname{Re}(\mathrm{Z}) / \operatorname{Im}(\mathrm{Z}): 1-160 k \Omega$. Changes of the analogue parameter need to be digitized. The measurements are done by an integrated readout based on the ADUCM355 sensing interface, a state-of-the-art combination of analogue circuitry with a micro-controller. The current and target specification of the $\mathrm{CO}_{2}$ sensor are given in Table VII for a $2.8 \mathrm{~V}$ supply.

8) Image sensor: The architecture of the full ultra-low power image sensor is depicted in Figure 3. The chip includes three main parts: the first is a digital pixel sensor (DPS) array that features all the pixels, row drivers and column buffers; the second is a controller that has two major roles: generating the different control signals to read the DPS array and transforming the time-encoded values of the pixels into digital-encoded values via a set of counters in the digital column readout; the third includes the peripherals that generate a clock for the counter, a ramp for the in-pixel comparator and bias voltages.

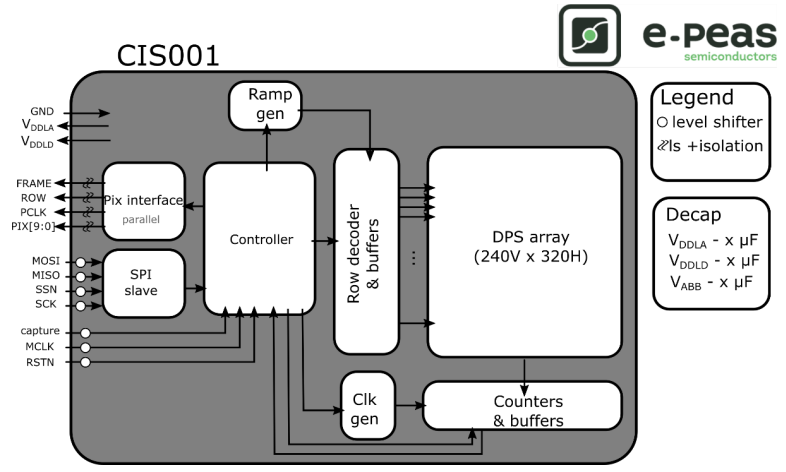

Fig. 3. Architecture of the ultra-low power QVGA time-based CMOS image sensor

Table VIII gives the current and target specifications of the image sensor. In the target design, emphasis will be put on the reduction of the energy consumption, both in active and sleep mode. The resolution will be limited to QVGA to fit the mechanical constraints and to limit the power consumption of the sensor.

9) Wireless communication: The data collected by the card should be transferred to the user or other devices for further processing. This will be performed by using different forms of wireless communication, especially protocols that are readily available. Since the communication is likely to impose a significant energy load, it is important to use appropriate protocols and optimize the energy consumption without penalizing the functionality of the ASSC. For that reason, two different modules are considered; a short-range and a long-range one. For short-range RF, the BLE protocol will be used, as it is optimized on lower power consumption. The RSL10 [12] wireless module offers the lowest power consumption in terms of current draw and already has a BLE stack implemented, therefore it is the SoC that will be used for the AMANDA ASSC. For long-range communication, LoRA will be used, as it can provide data transmission in distances over $15 \mathrm{~km}$ with minimal power consumption. For the ASSC, the SX1261 [13] 
TABLE VII

SPECIFICATIONS OF THE $\mathrm{CO}_{2}$ SENSOR

\begin{tabular}{|c|c|c|c|c|c|c|}
\hline COO $_{2}$ sensor & \multicolumn{3}{|c|}{ Current } & \multicolumn{3}{c|}{ Target } \\
\hline & Wake-up & Measurement & Sleep & Wake-up & Measurement & Sleep \\
\hline Time & $200 \mathrm{~ms}$ & $500 \mathrm{~ms}$ & - & $180 \mathrm{~ms}$ & $250 \mathrm{~ms}$ & - \\
\hline Electrical current & $4 \mathrm{~mA}$ & $20 \mathrm{~mA}$ & $9 \mu \mathrm{A}$ & $4 \mathrm{~mA}$ & $8 \mathrm{~mA}$ & $3 \mu \mathrm{A}$ \\
\hline Power & $2.2 \mathrm{~mW}$ & $28 \mathrm{~mW}$ & - & $2 \mathrm{~mW}$ & $5.6 \mathrm{~mW}$ & - \\
\hline
\end{tabular}

long range low power wireless transceiver will be utilized, as preliminary testing showed that it has excellent performance in terms of energy consumption and range for outdoor usage.

TABLE VIII

SPECIFICATIONS OF THE IMAGE SENSOR

\begin{tabular}{|c|c|c|}
\hline Image sensor & Current & Target \\
\hline Technology & $0.18 \mu \mathrm{m}$ CMOS & $0.18 \mu \mathrm{m}$ CIS \\
\hline Array resolution & $640 \mathrm{x} 480(\mathrm{VGA})$ & $320 \mathrm{x} 240(\mathrm{QVGA})$ \\
\hline Max frame rate & 8.5 & 30 \\
\hline Pixel size & $5.8 \times 5.8 \mu \mathrm{m}^{2}$ & $4.8 \times 4.8 \mu \mathrm{m}^{2}$ \\
\hline Dynamic range & $60 \mathrm{~dB}$ & $80 \mathrm{~dB}$ \\
\hline Peak SNR & $37.5 \mathrm{~dB}$ & $45 \mathrm{~dB}$ \\
\hline Energy & $88 \mathrm{pJ} / \mathrm{pixel} / \mathrm{frame}$ & $50 \mathrm{pJ} / \mathrm{pixel} /$ frame \\
\hline
\end{tabular}

10) Peripherals: For memory, the $8 k B$ MB85RS64TU FRAM component from Fujitsu will be used. Several variations with different memory sizes and serial interfaces (SPI or $\mathrm{I}^{2} \mathrm{C}$ ) exist. Consequently, the appropriate version will be chosen, depending on the use scenario. The RV-3028-C7 RTC will also be integrated to the ASSC, an SMT RTC module from Micro Crystal. It incorporates an integrated CMOS circuit together with a crystal. The device operates under vacuum in a hermetically sealed ceramic package with a metal lid. It has an $\mathrm{I}^{2} \mathrm{C}$ interface. Finally, the TPL5110-Q1 nano-timer will be used. The device is a low power timer with an integrated metaloxide-semiconductor field-effect transistor driver, ideal for power gating in duty cycled or battery powered applications. It can enable the power supply line and drastically reduce the overall system stand by current during the sleep time. Such power savings enable the use of significantly smaller energy storage units making it well suited for energy harvesting or wireless sensor applications.

\section{CONCLUSiOnS}

This paper introduced AMANDA, an embedded autonomous sensing system that will consist of a number of components such as PV harvesters, energy storage and power management units, MCUs and sensors, all packed with a form factor under $3 \mathrm{~mm}$ in thickness. AMANDA will introduce technical breakthroughs that will boost further miniaturization, offer a small footprint and ultra-low power consumption allowing for both indoors and outdoors operation.

Updates on the process and technical challenges of the development, optimization and integration of the system components, the testing and optimization of the system as well as of the evaluation of the AMANDA ASSC, will be subsequently reported as the project progresses.

\section{ACKNOWLEDGMENT}

This work is co-funded by the European Union (EU) within the AMANDA project under grant agreement number 825464. The AMANDA project is part of the EU Framework Programme for Research and Innovation Horizon 2020.

\section{REFERENCES}

[1] MordorIntelligence, "Smart sensors market - growth, trends, and forecast (2019 - 2024)." [Online]. Available: https://www.mordorintelligence. com/industry-reports/global-smart-sensors-market-industry

[2] C.-T. Lee, Y.-H. Liang, P. H. Chou, A. H. Gorji, S. M. Safavi, W.C. Shih, and W.-T. Chen, "Ecomicro: a miniature self-powered inertial sensor node based on bluetooth low energy," in Proceedings of the International Symposium on Low Power Electronics and Design. ACM, 2018, p. 30.

[3] W. Gao, S. Emaminejad, H. Y. Y. Nyein, S. Challa, K. Chen, A. Peck, H. M. Fahad, H. Ota, H. Shiraki, D. Kiriya et al., "Fully integrated wearable sensor arrays for multiplexed in situ perspiration analysis," Nature, vol. 529, no. 7587, p. 509, 2016.

[4] T. Choudhury, G. Borriello, S. Consolvo, D. Haehnel, B. Harrison, B. Hemingway, J. Hightower, K. Koscher, A. LaMarca, J. A. Landay et al., "The mobile sensing platform: An embedded activity recognition system," IEEE Pervasive Computing, vol. 7, no. 2, pp. 32-41, 2008.

[5] Y. Yang, K. Xu, T. Vervust, and J. Vanfleteren, "Multifunctional and miniaturized flexible sensor patch: Design and application for in situ monitoring of epoxy polymerization," Sensors and Actuators B: Chemical, vol. 261, pp. 144-152, 2018.

[6] M. Meli and L. Hegetschweiler, "Affordable energy autonomous wireless sensor for day and night," in Embedded World Conference, Nuremberg, February 23-25, 2016. WEKA Fachmedien, 2016.

[7] A. Gomez, L. Sigrist, M. Magno, L. Benini, and L. Thiele, "Dynamic energy burst scaling for transiently powered systems," in Proceedings of the 2016 Conference on Design, Automation \& Test in Europe. EDA Consortium, 2016, pp. 349-354.

[8] M. Meli and M. Da Silva, "Battery-less sensor nodes for 802.15. 4/zigbee wireless networks," in Wireless Congress: Systems \& Applications, Munich, November 9-10, 2011. Weka, 2011.

[9] N. L. Ismail, M. Kassim, M. Ismail, and R. Mohamad, "A review of low power wide area technology in licensed and unlicensed spectrum for iot use cases," Bulletin of Electrical Engineering and Informatics, vol. 7, no. 2, pp. 183-190, 2018.

[10] M. Brütsch, C. Brülisauer, L. Widmer, R. Kräuchi, D. Truninger, and M. Meli, "Comparing the energy requirements of bluetooth smart devices (november 2018)," in Wireless Congress Proceedings. WEKA, 2018.

[11] Microdul, "Ultra-low power, two channel capacitive sensor and touch switch for human body detection." [Online]. Available: https://www.microdul.com/en/assets/public/images/content/ MS8891A_Datasheet_M90-32-0555_EN.pdf

[12] OnSemiconductor, "Bluetooth 5 radio system-on-chip (soc)." [Online]. Available: https://www.onsemi.com/pub/Collateral/RSL10-D.PDF

[13] Semtech, "Sx1261/2 long range, low power, sub-ghz rf transceiver. rev. 1.1." [Online]. Available: https://www.semtech.com/uploads/documents/ DS_SX1261-2_V1.1.pdf 